\title{
A Two-Parameter Family of Fourth-Order Iterative Methods with Optimal Convergence for Multiple Zeros
}

\author{
Young Ik Kim and Young Hee Geum \\ Department of Applied Mathematics, Dankook University, Cheonan 330-714, Republic of Korea \\ Correspondence should be addressed to Young Hee Geum; conpana@empal.com
}

Received 25 October 2012; Revised 2 December 2012; Accepted 19 December 2012

Academic Editor: Fazlollah Soleymani

Copyright ( 2013 Y. I. Kim and Y. H. Geum. This is an open access article distributed under the Creative Commons Attribution License, which permits unrestricted use, distribution, and reproduction in any medium, provided the original work is properly cited.

We develop a family of fourth-order iterative methods using the weighted harmonic mean of two derivative functions to compute approximate multiple roots of nonlinear equations. They are proved to be optimally convergent in the sense of Kung-Traub's optimal order. Numerical experiments for various test equations confirm well the validity of convergence and asymptotic error constants for the developed methods.

\section{Introduction}

A development of new iterative methods locating multiple roots for a given nonlinear equation deserves special attention on both theoretical and numerical interest, although prior knowledge about the multiplicity of the sought zero is required [1]. Traub [2] discussed the theoretical importance of multiple-root finders, although the multiplicity is not known a priori by stating: "since the multiplicity of a zero is often not known a priori, the results are of limited value as far as practical problems are concerned. The study is, however, of considerable theoretical interest and leads to some surprising results." This motivates our analysis for multiple-root finders to be shown in this paper. In case the multiplicity is not known, interested readers should refer to the methods suggested by Wu and Fu [3] and Yun $[4,5]$.

Various iterative schemes finding multiple roots of a nonlinear equation with the known multiplicity have been proposed and investigated by many researchers [6-12]. Neta and Johnson [13] presented a fourth-order method extending Jarratt's method. Neta [14] also developed a fourth-order method requiring one-function and three-derivative evaluations per iteration grounded on a Murakami's method [15]. Shengguo et al. [16] proposed the following fourth-order method which needs evaluations of one function and two derivatives per iteration for $x_{0}$ chosen in a neighborhood of the sought zero $\alpha$ of $f(x)$ with known multiplicity $m \geq 1$ as follows:

$$
\begin{array}{r}
x_{n+1}=x_{n}-\frac{\beta f^{\prime}\left(x_{n}\right)+\phi f^{\prime}\left(y_{n}\right)}{f^{\prime}\left(x_{n}\right)+\delta f^{\prime}\left(y_{n}\right)} \frac{f\left(x_{n}\right)}{f^{\prime}\left(x_{n}\right)}, \\
n=0,1,2, \ldots,
\end{array}
$$

where $y_{n}=x_{n}-(2 m /(m+2))\left(f\left(x_{n}\right) / f^{\prime}\left(x_{n}\right)\right), \beta=-\left(m^{2} / 2\right)$, $\phi=(1 / 2)\left(m(m-2) /(m /(m+2))^{m}\right)$, and $\delta=-(m /(m+2))^{-m}$ with the following error equation:

$$
e_{n+1}=K_{4} e_{n}^{4}+O\left(e_{n}^{5}\right), \quad n=0,1,2, \ldots,
$$

where $K_{4}=\left(\left(-2+2 m+2 m^{2}+m^{3}\right) / 3 m^{4}(m+1)^{3}\right) \theta_{1}^{3}-(1 / m(m+$ $\left.1)^{2}(m+2)\right) \theta_{1} \theta_{2}+\left(m /(m+2)^{3}(m+1)(m+3)\right) \theta_{3}, e_{n}=x_{n}-\alpha$, and $\theta_{j}=f^{(m+j)}(\alpha) / f^{(m)}(\alpha)$ for $j=1,2,3$.

Based on Jarratt [17] scheme for simple roots, J. R. Sharma and R. Sharma [18] developed the following fourth order of convergent scheme:

$$
\begin{aligned}
x_{n+1}=x_{n} & -A \frac{f\left(x_{n}\right)}{f^{\prime}\left(x_{n}\right)}-B \frac{f\left(x_{n}\right)}{f^{\prime}\left(y_{n}\right)} \\
& -C\left(\frac{f\left(x_{n}\right)}{f^{\prime}\left(y_{n}\right)}\right)^{2}\left(\frac{f\left(x_{n}\right)}{f^{\prime}\left(x_{n}\right)}\right)^{-1}, \quad n=0,1,2, \ldots,
\end{aligned}
$$


where $A=(1 / 8) m\left(m^{3}-4 m+8\right), B=-(1 / 4) m(m-1)(m+$ $2)^{2}(m /(m+2))^{m}, C=(1 / 8) m(m+2)^{3}(m /(m+2))^{2 m}$, and $y_{n}=x_{n}-(2 m /(m+2))\left(f\left(x_{n}\right) / f^{\prime}\left(x_{n}\right)\right)$ and derived the error equation below:

$$
\begin{aligned}
e_{n+1}= & \left\{\frac{-8+12 m+14 m^{2}+14 m^{3}+6 m^{4}+m^{5}}{3 m^{4}(m+2)^{2}} \mathscr{A}_{1}^{3}\right. \\
& \left.-\frac{1}{m} \mathscr{A}_{1} \mathscr{A}_{2}+\frac{m}{(m+2)^{2}} \mathscr{A}_{3}\right\} e_{n}^{4}+O\left(e_{n}^{5}\right),
\end{aligned}
$$

where $\mathscr{A}_{j}=(m ! /(m+j) !)\left(f^{(m+j)}(\alpha) / f^{(m)}(\alpha)\right)=(m ! /(m+$ j)!) $\theta_{j}$ for $j=1,2,3$.

The above error equation can be expressed in terms of $\theta_{j}(j=1,2,3)$ as follows:

$$
e_{n+1}=C_{4} e_{n}^{4}+O\left(e_{n}^{5}\right)
$$

where $C_{4}=\left(\left(-8+12 m+14 m^{2}+14 m^{3}+6 m^{4}+m^{5}\right) / 3 m^{4}(m+\right.$ $\left.1)^{3}(m+2)^{2}\right) \theta_{1}^{3}-\left(1 / m(m+1)^{2}(m+2)\right) \theta_{1} \theta_{2}+\left(m /(m+2)^{3}(m+\right.$ 1) $(m+3)) \theta_{3}$.

We now proceed to develop a new iterative method finding an approximate root $\alpha$ of a nonlinear equation $f(x)=$ 0 , assuming the multiplicity of $\alpha$ is known. To do so, we first suppose that a function $f: \mathbb{C} \rightarrow \mathbb{C}$ has a multiple root $\alpha$ with integer multiplicity $m \geq 1$ and is analytic in a small neighborhood of $\alpha$. Then we propose a new iterative method free of second derivatives below with an initial guess $x_{0}$ sufficiently close to $\alpha$ as follows:

$$
\begin{aligned}
x_{n+1}= & y_{n}-a \frac{f\left(x_{n}\right)}{f^{\prime}\left(x_{n}\right)}-b \frac{F\left(y_{n}\right)}{f^{\prime}\left(x_{n}\right)} \\
& -c \frac{f\left(x_{n}\right)}{f^{\prime}\left(y_{n}\right)}, \quad n=0,1,2, \ldots,
\end{aligned}
$$

where

$$
\begin{aligned}
y_{n} & =x_{n}-\gamma \frac{f\left(x_{n}\right)}{f^{\prime}\left(x_{n}\right)}, \\
F\left(y_{n}\right) & =f\left(x_{n}\right)+\left(y_{n}-x_{n}\right) \frac{\lambda f^{\prime}\left(x_{n}\right) f^{\prime}\left(y_{n}\right)}{f^{\prime}\left(x_{n}\right)+\rho f^{\prime}\left(y_{n}\right)},
\end{aligned}
$$

with $a, b, c, \gamma, \lambda$, and $\rho$ as parameters to be chosen for maximal order of convergence $[2,19]$. One should note that $F\left(y_{n}\right)$ is obtained from Taylor expansion of $f\left(y_{n}\right)$ about $x_{n}$ up to the first-order terms with weighted harmonic mean [20] of $f^{\prime}\left(x_{n}\right)$ and $f^{\prime}\left(y_{n}\right)$.

Theorem 1 shows that proposed method (6) possesses 2 free parameters $\lambda$ and $\rho$. A variety of free parameters $\lambda$ and $\rho$ give us an advantage that iterative scheme (6) can develop various numerical methods. One can often have a freedom to select best suited parameters $\lambda$ and $\rho$ for a sought zero $\alpha$. Several interesting choices of $\lambda$ and $\rho$ further motivate our current analysis. As seen in Table 1, we consider five kinds of methods $\mathrm{Y} 1, \mathrm{Y} 2, \mathrm{Y} 3, \mathrm{Y} 4$, and $\mathrm{Y} 5$ list selected parameters $(\lambda, \rho)$, and the corresponding values $(a, b, c)$, respectively.
If $\lambda=-\left(m((m+2) / m)^{m+2} /\left(m^{2}+2 m+4\right)\right)$ and $\rho=-((m+$ $2) / m)^{m}$ are selected, then we obtain $a=0, b=-\left(m\left(m^{2}+\right.\right.$ $2 m+4) / 2(m+2))$, and $c=0$, in which case iterative scheme (6) becomes method Y5 mentioned above and blackuces to iterative scheme (1) developed by Shengguo et al. [16].

In this paper, we investigate the optimal convergence of the fourth-order methods for multiple-root finders with known multiplicity in the sense of optimal order claimed by Kung-Traub [21] and derive the error equation. We find that our proposed schemes require one evaluation of the function and two evaluations of first derivative and satisfy the optimal order. In addition, through a variety of numerical experiments we wish to confirm that the proposed methods show well the convergence behavior predicted by the developed theory.

\section{Convergence Analysis}

In this section, we describe a choice of parameters $a, b$, and $c$ in terms of $\lambda$ and $\rho$ to get fourth-order convergence for our proposed scheme (6).

Theorem 1. Let $f: \mathbb{C} \rightarrow \mathbb{C}$ have a zero $\alpha$ with integer multiplicity $m \geq 1$ and be analytic in a small neighborhood of $\alpha$. Let $\kappa=(m /(m+2))^{m}, \theta_{j}=\left(f^{(m+j)}(\alpha) / f^{(m)}(\alpha)\right)$ for $j \epsilon$ $\mathbb{N}$. Let $x_{0}$ be an initial guess chosen in a sufficiently small neighborhood of $\alpha$. Let $a=(m /(m+2))\{m-(1 / 8) m(m+$ $2)^{3}(1+\kappa \rho)-(m+2)^{2}(m(-1+2 \kappa \lambda)-(m+2) \kappa \rho)(m+(m+$ 2) $\left.\kappa \rho)^{2} / 16 m \kappa \lambda\right\}, b=-\left((m+2)(m+(m+2) \kappa \rho)^{3} / 16 \kappa \lambda\right)$, $c=(1 / 8) m(m+2)^{3} \kappa(1+\kappa \rho)$, and $\gamma=2 m /(m+2)$. Let $\lambda, \rho \in \mathbb{R}$ be two free constant parameters. Then iterative method (6) is of order four and defines a two-parameter family of iterative methods with the following error equation:

$$
e_{n+1}=\psi_{4} e_{n}^{4}+O\left(e_{n}^{5}\right), \quad n=0,1,2, \ldots
$$

where $e_{n}=x_{n}-\alpha$ and

$$
\begin{aligned}
\psi_{4}= & \frac{8+2 m+6 m^{2}+4 m^{3}+m^{4}-(24 \kappa \rho /(m+(m+2) \kappa \rho))}{3 m^{4}(m+1)^{3}(m+2)} \theta_{1}^{3} \\
& -\frac{1}{m(m+1)^{2}(m+2)} \theta_{1} \theta_{2} \\
& +\frac{m}{(m+2)^{3}(m+1)(m+3)} \theta_{3} .
\end{aligned}
$$

Proof. Using Taylor's series expansion about $\alpha$, we thave the following relations:

$$
\begin{aligned}
f\left(x_{n}\right)= & \frac{f^{(m)}(\alpha)}{m !} e_{n}^{m} \\
& \times\left[1+A_{1} e_{n}+A_{2} e_{n}^{2}+A_{3} e_{n}^{3}+A_{4} e_{n}^{4}+O\left(e_{n}^{5}\right)\right],
\end{aligned}
$$




$$
\begin{aligned}
f^{\prime}\left(x_{n}\right)= & \frac{f^{(m)}(\alpha)}{(m-1) !} e_{n}{ }^{m-1} \\
& \times\left[1+B_{1} e_{n}+B_{2} e_{n}^{2}+B_{3} e_{n}^{3}+B_{4} e_{n}^{4}+O\left(e_{n}^{5}\right)\right],
\end{aligned}
$$

where $A_{k}=(m ! /(m+k) !) \theta_{k}, B_{k}=((m-1) ! /(m+k-1) !) \theta_{k}$, and $\theta_{k}=\left(f^{(m+k)}(\alpha) / f^{(m)}(\alpha)\right)$ for $k \in \mathbb{N}$.

Dividing (10) by (11), we obtain

$$
\frac{f\left(x_{n}\right)}{f^{\prime}\left(x_{n}\right)}=\frac{1}{m}\left[e_{n}-K_{1} e_{n}^{2}-K_{2} e_{n}^{3}+K_{3} e_{n}^{4}+O\left(e_{n}^{5}\right)\right],
$$

where $K_{1}=-A_{1}+B_{1}, K_{2}=-A_{2}+A_{1} B_{1}-B_{1}^{2}+B_{2}$, and $K_{3}=-A_{3}+A_{2} B_{1}-A_{1} B_{1}^{2}+B_{1}^{3}+A_{1} B_{2}-2 B_{1} B_{2}+B_{3}$.

Expressing $\gamma=m(1-t)$ in terms of a new parameter $t$ for algebraic simplicity, we get

$$
\begin{aligned}
y_{n}= & x_{n}-\gamma \frac{f\left(x_{n}\right)}{f^{\prime}\left(x_{n}\right)} \\
= & \alpha+t e_{n}+K_{1}(1-t) e_{n}^{2} \\
& +K_{2}(1-t) e_{n}^{3}+K_{3}(1-t) e_{n}^{4}+O\left(e_{n}^{5}\right) .
\end{aligned}
$$

Since $f^{\prime}\left(y_{n}\right)$ can be expressed from $f^{\prime}\left(x_{n}\right)$ in (11) with $e_{n}$ substituted by $\left(y_{n}-\alpha\right)$ from (13), we get

$$
\begin{aligned}
& f^{\prime}\left(y_{n}\right) \\
& =\frac{f^{(m)}(\alpha)}{(m-1) !} e_{n}^{m-1} \\
& \times\left[t^{m-1}+t^{m-2}\left[B_{1} t^{2}-K_{1}(m-1)(t-1)\right] e_{n}+t^{m-3}\right. \\
& \times \frac{K_{1}^{2}(m-2)(m-1)(t-1)^{2}-2 m B_{1} K_{1}(t-1) t^{2}+2 t\left[B_{2} t^{3}-K_{2}(m-1)(t-1)\right]}{2} \\
& \times e_{n}^{2}+\frac{t^{m-4}}{6}\left\{-K_{1}^{3}(m-3)(m-2)(m-1)(t-1)^{3}\right. \\
& \quad+3 B_{1} K_{1}^{2}(m-1) m(t-1)^{2} t^{2}-6 K_{1} t(t-1) \\
& \quad \times\left[-K_{2}(m-2)(m-1)(t-1)+B_{2}(m+1) t^{3}\right] \\
& \left.\left.\quad-6 t^{2}\left[B_{1} K_{2} m t(t-1)-B_{3} t^{4}+K_{3}(m-1)(t-1)\right]\right\} e_{n}^{3}\right]
\end{aligned}
$$

$+O\left(e_{n}^{4}\right)$.

With the aid of symbolic computation of Mathematica [22], we substitute (10)-(14) into proposed method (6) to obtain the error equation as

$$
\begin{aligned}
e_{n+1} & =y_{n}-\alpha-a \frac{f\left(x_{n}\right)}{f^{\prime}\left(x_{n}\right)}-b \frac{F\left(y_{n}\right)}{f^{\prime}\left(x_{n}\right)}-c \frac{f\left(x_{n}\right)}{f^{\prime}\left(y_{n}\right)} \\
& =\psi_{1} e_{n}+\psi_{2} e_{n}^{2}+\psi_{3} e_{n}^{3}+\psi_{4} e_{n}^{4}+O\left(e_{n}^{5}\right),
\end{aligned}
$$

where $\psi_{1}=t-\left(\left(a+b+c t^{1-m}\right) / m\right)-\left(b(t-1) t^{m-1} \lambda /\left(1+t^{m-1} \rho\right)\right)$, and the coefficient $\psi_{i}(i=2,3,4)$ may depend on parameters $t, a, b, c, \lambda$, and $\rho$.
Solving $\psi_{1}=0$ and $\psi_{2}=0$ for $a$ and $b$, respectively, we get after simplifications

$$
\begin{aligned}
& a=-b+t\left(m-c t^{-m}\right)-\frac{b m(t-1) t^{m-1} \lambda}{1+t^{m-1} \rho}, \\
& b=\frac{t^{2-2 m}\left[m t^{m}+c(t-1)(1+m(t-1)+t)\right]\left(1+t^{m-1} \rho\right)^{2}}{m(t-1)^{2}(1+m(t-1)+t) \lambda} .
\end{aligned}
$$

Putting $\psi_{3}=\psi_{31} \theta_{1}^{2}+\psi_{32} \theta_{2}$, we have

$$
\begin{aligned}
& \psi_{31}= \\
& \frac{t^{-1-m}\left(-2 c(t-1)^{2} t(1+m(t-1)+t)^{3}+m t^{m}\left(P_{1}+t^{m} P_{2} \rho\right)\right)}{2 m^{3}(m+1)^{2}(1+m(t-1)+t)\left(t+t^{m} \rho\right)} \\
& \psi_{32}=\frac{t(m-(m+2) t)}{m(m+1)(m+2)(1+m(t-1)+t)}
\end{aligned}
$$

where $P_{1}=t\left[2-3 m+m^{2}+\left(2+m-3 m^{2}\right) t+2(m+1)^{2} t^{2}\right]$ and $P_{2}=2 t^{2}(2+t)+m^{2}(t-1)[1+2(t-1) t]+m\left(1-3 t+4 t^{3}\right)$.

Observe that $\psi_{32}=0$ is satisfied with $t=m /(m+2)$. Solving $\psi_{31}=0$ for $c$, we get

$$
c=\frac{t^{m-1} m\left(P_{1}+t^{m} P_{2} \rho\right)}{2(t-1)^{2}(1+m(t-1)+t)^{3}} .
$$

Substituting $t=m /(m+2)$ into (16) and (19) with $\kappa=$ $(m /(m+2))^{m}$, we can rearrange these expressions to obtain

$$
\begin{aligned}
a=\frac{m}{m+2}\{m & -\frac{m(m+2)^{3}(1+\kappa \rho)}{8} \\
- & \left.\frac{(m+2)^{2}(m(-1+2 \kappa \lambda)-(m+2) \kappa \rho)(m+(m+2) \kappa \rho)^{2}}{16 m \kappa \lambda}\right\} \\
b & =-\frac{(m+2)(m+(m+2) \kappa \rho)^{3}}{16 \kappa \lambda} \\
c & =\frac{1}{8} m(m+2)^{3} \kappa(1+\kappa \rho) .
\end{aligned}
$$

Calculating by the aid of symbolic computation of Mathematica [22], we arrive at the error equation below:

$$
e_{n+1}=\psi_{4} e_{n}^{4}+O\left(e_{n}^{5}\right)
$$

where $\psi_{4}=\left(\left(8+2 m+6 m^{2}+4 m^{3}+m^{4}-(24 \kappa \rho /(m+(m+\right.\right.$ 2) $\left.\kappa \rho)) / 3 m^{4}(m+1)^{3}(m+2)\right) \theta_{1}^{3}-\left(1 / m(m+1)^{2}(m+2)\right) \theta_{1} \theta_{2}$ $+\left(m /(m+2)^{3}(m+1)(m+3)\right) \theta_{3}$ with $\kappa=(m /(m+2))^{m}$.

It is interesting to observe that error equation (23) has only one free parameter $\rho$, being independent of $\lambda$. Table 1 shows typically chosen parameters $\lambda$ and $\rho$ and defines various methods $Y_{k},(k=1,2, \ldots, 5)$ derived from (6). Method Y5 results in the iterative scheme (1) that Shengguo et al. [16] suggested. 
TABLE 1: Various methods with typical choice of parameters $(\lambda, \rho, a, b$, and $c)$.

$$
\begin{gathered}
a=\frac{m}{m+2}\left\{m+\frac{1}{8} m(m+2)^{3}(5 \kappa-1)-\frac{(m+2)^{2}}{16 m \kappa} \phi_{1}\right\} \\
\phi_{1}=(m-5(m+2) \kappa)^{2}(10 \kappa+m(7 \kappa-1)) \\
b=-\frac{(m+2)(m-5(m+2) \kappa)^{3}}{16 \kappa}, \\
c=\frac{1}{8} m(m+2)^{3} \kappa(1-5 \kappa)
\end{gathered}
$$

$$
\begin{gathered}
a=\frac{m}{m+2}\left\{m+\frac{1}{8} m(m+2)^{3}(3 \kappa-1)-\frac{(m+2)^{2}}{16 m \kappa} \phi_{2}\right\} \\
\phi_{2}=(m-3(m+2) \kappa)^{2}(6 \kappa+m(5 \kappa-1)), \\
b=-\frac{(m+2)(m-3(m+2) \kappa)^{3}}{16 \kappa}, \\
c=\frac{1}{8} m(m+2)^{3} \kappa(1-3 \kappa)
\end{gathered}
$$

$$
\begin{gathered}
a=\frac{m}{m+2}\left\{m-\frac{1}{8} m(m+2)^{3}(10 \kappa+1)-\frac{(m+2)^{2}}{16 m \kappa} \phi_{3}\right\} \\
\phi_{3}=(m+20 \kappa+8 m \kappa)(m+10(m+2) \kappa)^{2}, \\
b=-\frac{(m+2)(m+10(m+2) \kappa)^{3}}{16 \kappa}, \\
c=\frac{1}{8} m(m+2)^{3} \kappa(1+10 \kappa)
\end{gathered}
$$

\section{Numerical Examples and Conclusion}

In this section, we have performed numerical experiments using Mathematica Version 5 program to convince that the optimal order of convergence is four and the computed asymptotic error constant $\left|e_{n+1} / e_{n}^{4}\right|$ agrees well with the theoretical value $\eta$. To achieve the specified sufficient accuracy and to handle small number divisions appearing in asymptotic error constants, we have assigned 300 as the minimum number of digits of precision by the command $\$$ MinPrecision $=300$ and set the error bound $\epsilon$ to $10^{-250}$ for $\left|x_{n}-\alpha\right|<\epsilon$. We have chosen the initial values $x_{0}$ close to the sought zero $\alpha$ to get fourth-order convergence. Although computed values of $x_{n}$ are truncated to be accurate up to 250 significant digits and the inexact value of $\alpha$ is approximated to be accurate enough about up to 400 significant digits (with the command FindRoot $\left[f[x],\left\{x, x_{0}\right\}\right]$, PrecisionGoal $\rightarrow$ 400 , and WorkingPrecision $\rightarrow 600$ ), we list them up to 15 significant digits because of the limited space.

As a first example with a double zero $\alpha=\sqrt{3}$ and an initial guess $x_{0}=1.58$, we select a test function $f(x)=$ $\cos \left(\pi x^{2} / 6\right) \log \left(x^{2}-\sqrt{3} x+1\right)$. As a second experiment, we take another test function $f(x)=\left(16+x^{2}\right)^{3}\left(\log \left(x^{2}+17\right)\right)^{4}$ with a root $\alpha=-4 i$ of multiplicity $m=7$ and with an initial value $x_{0}=-3.94 i$.

Taking another test function $f(x)=\left(1-\sin \left(x^{2}\right)\right)$ $\left(\log \left(2 x^{2}-\pi+1\right)\right)^{4}$ with a $\operatorname{root} \alpha=-\sqrt{\pi / 2}$ of multiplicity $m=6$, we select $x_{0}=-1.18$ as an initial value.

Throughout these examples, we confirm that the order of convergence is four and the computed asymptotic error constant $\left|e_{n+1} / e_{n}^{4}\right|$ approaches well the theoretical value $\eta$. The 
TABLE 2: Convergence behavior with $f(x)=\cos \left(\pi x^{2} / 6\right) \log \left(x^{2}-\sqrt{3} x+1\right),(m, \lambda, \rho)=(2,5,5), \alpha=\sqrt{3}$.

\begin{tabular}{ccccc}
\hline$n$ & $x_{n}$ & $\left|f\left(x_{n}\right)\right|$ & $\left|x_{n}-\alpha\right|$ & $\left|e_{n+1} / e_{n}^{4}\right|$ \\
\hline 0 & 1.58 & 0.0716114 & 0.152051 & \\
1 & 1.73207355929649 & $1.62622 \times 10^{-9}$ & 0.0000227517 & 0.04256566818 \\
2 & 1.73205080756888 & $1.04639 \times 10^{-40}$ & $5.77127 \times 10^{-21}$ & 0.02153842658 \\
3 & 1.73205080756888 & $1.79209 \times 10^{-165}$ & $2.38839 \times 10^{-83}$ & 0.02152876768 \\
4 & 1.73205080756888 & $0.0 \times 10^{-599}$ & $0.0 \times 10^{-299}$ & \\
\hline
\end{tabular}

TABLE 3: Convergence behavior with $f(x)=\left(16+x^{2}\right)^{3}\left(\log \left(x^{2}+17\right)\right)^{4},(m, \lambda, \rho)=(7,1,-1), \alpha=-4 i$.

\begin{tabular}{ccccc}
\hline$n$ & $x_{n}$ & $\left|f\left(x_{n}\right)\right|$ & $\left|x_{n}-\alpha\right|$ & $\left|e_{n+1} / e_{n}^{4}\right|$ \\
\hline 0 & $-3.94 i$ & 0.00249127 & 0.0600000 & \\
1 & $-4.00000168928648 i$ & $8.23314 \times 10^{-35}$ & $1.68929 \times 10^{-6}$ & 0.1303461794 \\
2 & $-4.00000000000000 i$ & $2.27416 \times 10^{-160}$ & $1.95318 \times 10^{-24}$ & 0.2398437513 \\
3 & $-4.00000000000000 i$ & $1.32326 \times 10^{-662}$ & $3.49027 \times 10^{-96}$ & 0.2398240851 \\
4 & $-4.00000000000000 i$ & $0.0 \times 10^{-2096}$ & $0.0 \times 10^{-299}$ & \\
\hline
\end{tabular}

order of convergence and the asymptotic error constant are clearly shown in Tables 2,3, and 4 reaching a good agreement with the theory developed in Section 2.

The additional test functions $f_{1}, f_{2}, \ldots, f_{7}$ listed below further confirm the convergence behavior of our proposed method (6).

$$
\begin{gathered}
f_{1}(x)=\frac{x^{7}-x^{2}-7}{x^{5}+\sin x}, \quad \alpha=1.3657, \quad m=1, \\
x_{0}=1.32, \\
f_{2}(x)=\left(e^{x^{5}-x^{2}-7}-1\right)\left(7+x^{2}-x^{5}\right), \quad \alpha=-1.16-0.95 i, \\
m=2, \quad x_{0}=-1.14-0.92 i, \\
f_{3}(x)=\left(x^{6}-8\right)^{2} \log \left(x^{6}-7\right), \quad \alpha=\sqrt{2}, \quad m=3, \\
x_{0}=1.39, \\
f_{4}(x)=\left(3-x+x^{2}\right)^{4} \cot \left(x^{2}+1\right), \quad \alpha=\frac{1-\sqrt{11} i}{2}, \\
f_{5}(x)=\left(x^{4}-9 x^{3}+4 x^{2}-33 x-27\right)(\log (x-8))^{3}, \\
\alpha=9, \quad m=5, \quad x_{0}=8.79, \\
f_{6}(x)=(\log (1-\pi+x))^{6}, \quad \alpha=\pi, \quad m=6, \\
\alpha=-3, \quad m=7, \quad x_{0}=-2.88 .
\end{gathered}
$$

Table 5 shows the convergence behavior of $\left|x_{n}-\alpha\right|$ among methods S, J, Y1, Y2, Y3, and Y4, where S denotes the method proposed by Shengguo et al. [16], J the method proposed by J. R. Sharma and R. Sharma [18], the methods Y1 to Y4 are described in Table 1. It is certain that proposed method (6) needs one evaluation of the function $f$ and two evaluations of the first derivative $f^{\prime}$ per iteration. Consequently, the corresponding efficiency index [2] is found to be $4^{1 / 3} \approx 1.587$, which is optimally consistent with the conjecture of KungTraub [21]. For the particularly chosen test functions in these numerical experiments, methods Y1 to Y4 have shown better accuracy than methods $S$ and J.

Nevertheless, the favorable performance of proposed scheme (6) is not always expected since no iterative method always shows best accuracy for all the test functions. If we look at the asymptotic error equation $\eta=\eta(f, \alpha, p)=$ $\lim _{n \rightarrow \infty}\left|\left(x_{n+1}-\alpha\right) /\left(x_{n}-\alpha\right)^{p}\right|$ closely, we should note that the computational accuracy is sensitively dependent on the structures of the iterative methods, the sought zeros, convergence orders, the test functions, and good initial values.

It is important to properly choose enough number of precision digits. If $e_{k}$ is small, $e_{k}^{p}$ gets much smaller, as $k$ increases. If the number of precision is small and error bound $\epsilon$ is not small enough, the term $e_{k}^{p}$ causes a great loss of significant digits due to magnified round-off errors. This hinders us from verifying $p$ and $\eta$ more accurately.

Bold-face numbers in Table 5 refer to the least error until the prescribed error bound is met. This paper has confirmed optimal fourth-order convergence proved the correct error equation for proposed iterative methods (6), using the weighted harmonic mean of two derivatives to find approximate multiple zeros of nonlinear equations. We remark that the error equation of (6) contains only one free parameter $\rho$, being independent of $\lambda$.

We still further need to discuss some aspects of root finding for ill-conditioned problems as well as sensitive dependence of zeros on initial values for iterative methods. As is well known, a high-degree (say, degree higher than 20, taking the multiplicity of a zero into account) polynomial is very likely to be ill conditioned. In this case, small changes in the coefficients can greatly alter the zeros. The small 
TABLE 4: Convergence behavior with $f(x)=\left(1-\sin \left(x^{2}\right)\right)\left(\log \left(2 x^{2}-\pi+1\right)\right)^{4},(m, \lambda, \rho)=(6,1,-1), \alpha=-\sqrt{\pi / 2}$.

\begin{tabular}{ccccc}
\hline$n$ & $x_{n}$ & $\left|f\left(x_{n}\right)\right|$ & $\left|x_{n}-\alpha\right|$ & $\left|e_{n+1} / e_{n}^{4}\right|$ \\
\hline 0 & -1.18 & 0.000601837 & 0.0733141 & \\
1 & -1.25334379618481 & $1.35039 \times 10^{-24}$ & 0.0000296589 & 1.026605711 \\
2 & -1.25331413731550 & $7.41245 \times 10^{-109}$ & $2.68363 \times 10^{-19}$ & 0.3468196331 \\
3 & -1.25331413731550 & $6.74576 \times 10^{-446}$ & $1.79984 \times 10^{-75}$ & 0.3470127318 \\
4 & -1.25331413731550 & $4.62631 \times 10^{-1794}$ & $3.64139 \times 10^{-300}$ & \\
\hline
\end{tabular}

TABLE 5: Comparison of $\left|x_{n}-\alpha\right|$ for high-order iterative methods.

\begin{tabular}{|c|c|c|c|c|c|c|c|c|}
\hline$f(x)$ & $x_{0}$ & $\left|x_{n}-\alpha\right|$ & $\mathrm{S}$ & $\mathrm{J}$ & Y1 & $\mathrm{Y} 2$ & Y3 & Y4 \\
\hline \multirow{4}{*}{$f_{1}$} & \multirow{4}{*}{1.32} & $\left|x_{1}-\alpha\right|$ & $5.37 e-7^{\dagger}$ & $4.00 e-7$ & $6.26 e-8$ & $5.37 e-7$ & $1.96 e-7$ & $2.14 e-6$ \\
\hline & & $\left|x_{2}-\alpha\right|$ & $1.20 e-26$ & $1.27 e-27$ & $7.31 e-31$ & $1.2 e-26$ & $9.24 e-30$ & $9.29 e-24$ \\
\hline & & $\left|x_{3}-\alpha\right|$ & $3.02 e-105$ & $1.27 e-109$ & $1.36 \mathrm{e}-122$ & $3.02 e-104$ & $4.53 e-119$ & $3.24 e-93$ \\
\hline & & $\left|x_{4}-\alpha\right|$ & $0 . e-299$ & $0 . e-299$ & $0 . e-299$ & $0 . e-299$ & $0 . e-299$ & $0 . e-299$ \\
\hline \multirow{5}{*}{$f_{2}$} & \multirow{5}{*}{$-1.14-0.92 i$} & $\left|x_{1}-\alpha\right|$ & 0.001168 & 0.00144 & 0.00124 & 0.00097 & 0.00136 & 0.0018 \\
\hline & & $\left|x_{2}-\alpha\right|$ & $4.99 e-10$ & $1.60 e-9$ & $7.38 e-10$ & $1.45 e-10$ & $1.19 e-9$ & $5.18 e-9$ \\
\hline & & $\left|x_{3}-\alpha\right|$ & $1.65 e-35$ & $2.46 e-33$ & $8.97 e-35$ & $7.24 e-38$ & $7.15 e-34$ & $3.46 e-31$ \\
\hline & & $\left|x_{4}-\alpha\right|$ & $2.02 e-137$ & $1.37 e-128$ & $1.95 e-134$ & $4.44 \mathrm{e}-147$ & $9.06 e-131$ & $6.89 e-120$ \\
\hline & & $\left|x_{5}-\alpha\right|$ & $0 . e-299$ & $0 . e-299$ & $0 . e-299$ & $0 . e-299$ & $0 . e-299$ & $0 . e-299$ \\
\hline \multirow{5}{*}{$f_{3}$} & \multirow{5}{*}{1.39} & $\left|x_{1}-\alpha\right|$ & 0.000428741 & 0.0002722 & 0.000399 & 0.00492273 & 0.0003078 & 0.000191074 \\
\hline & & $\left|x_{2}-\alpha\right|$ & $1.77 e-12$ & $3.38 e-13$ & $1.37 e-12$ & $5.14 e-8$ & $5.32 e-13$ & $9.02 e-14$ \\
\hline & & $\left|x_{3}-\alpha\right|$ & $5.19 e-46$ & $8.05 e-49$ & $1.89 e-46$ & $1.19 e-28$ & $4.73 e-48$ & $4.48 e-51$ \\
\hline & & $\left|x_{4}-\alpha\right|$ & $3.79 e-180$ & $2.57 e-191$ & $6.92 e-182$ & $3.46 e-111$ & $2.96 e-188$ & $2.73 e-200$ \\
\hline & & $\left|x_{5}-\alpha\right|$ & $0 . e-299$ & $0 . e-299$ & $0 . e-299$ & $0 . e-299$ & $0 . e-299$ & $0 . e-299$ \\
\hline \multirow{5}{*}{$f_{4}$} & \multirow{5}{*}{$0.47-1.79 i$} & $\left|x_{1}-\alpha\right|$ & 0.00016 & 0.000159 & 0.00016 & 0.000153 & 0.0001602 & 0.000159 \\
\hline & & $\left|x_{2}-\alpha\right|$ & $3.55 e-16$ & $3.39 e-16$ & $3.55 e-16$ & $2.81 e-16$ & $3.43 e-16$ & $3.32 e-16$ \\
\hline & & $\left|x_{3}-\alpha\right|$ & $8.36 e-63$ & $6.94 e-63$ & $8.42 e-63$ & $3.14 e-63$ & $7.28 e-63$ & $6.33 e-63$ \\
\hline & & $\left|x_{4}-\alpha\right|$ & $2.56 e-249$ & $1.21 e-249$ & $2.63 e-249$ & $4.89 e-249$ & $1.46 e-249$ & $8.37 e-250$ \\
\hline & & $\left|x_{5}-\alpha\right|$ & $0 . e-299$ & $0 . e-299$ & $0 . e-299$ & $0 . e-299$ & $0 . e-299$ & $0 . e-299$ \\
\hline \multirow{4}{*}{$f_{5}$} & \multirow{4}{*}{8.79} & $\left|x_{1}-\alpha\right|$ & 0.0000222 & 0.0000184 & 0.000023 & 0.0000118 & 0.0000193 & 0.0000164 \\
\hline & & $\left|x_{2}-\alpha\right|$ & $1.43 e-21$ & $5.53 e-22$ & $1.90 e-21$ & $5.51 e-23$ & $7.12 e-22$ & $3.06 e-22$ \\
\hline & & $\left|x_{3}-\alpha\right|$ & $2.47 e-86$ & $4.46 e-88$ & $8.07 e-86$ & $2.60 e-92$ & $1.29 e-87$ & $3.71 e-89$ \\
\hline & & $\left|x_{4}-\alpha\right|$ & $0 . e-299$ & $0 . e-299$ & $0 . e-299$ & $0 . e-299$ & $0 . e-299$ & $0 . e-299$ \\
\hline \multirow{4}{*}{$f_{6}$} & \multirow{4}{*}{3.09} & $\left|x_{1}-\alpha\right|$ & $2.84 e-7$ & $3.45 e-7$ & $2.36 e-7$ & $4.14 e-7$ & $3.30 e-7$ & $3.65 e-7$ \\
\hline & & $\left|x_{2}-\alpha\right|$ & $2.51 e-28$ & $6.55 e-28$ & $1.00 e-28$ & $1.62 e-27$ & $5.28 e-28$ & $8.68 e-28$ \\
\hline & & $\left|x_{3}-\alpha\right|$ & $1.52 e-112$ & $8.50 e-111$ & $3.24 \mathrm{e}-114$ & $3.86 e-109$ & $3.44 e-111$ & $2.76 e-110$ \\
\hline & & $\left|x_{4}-\alpha\right|$ & $0 . e-299$ & $0 . e-299$ & $0 . e-299$ & $0 . e-299$ & $0 . e-299$ & $0 . e-299$ \\
\hline \multirow{5}{*}{$f_{7}$} & \multirow{5}{*}{-2.88} & $\left|x_{1}-\alpha\right|$ & 0.00109 & 0.001241 & 0.00088 & 0.00137 & 0.0012 & 0.00128 \\
\hline & & $\left|x_{2}-\alpha\right|$ & $2.04 e-11$ & $1.97 e-11$ & $3.14 e-11$ & $1.00 e-10$ & $6.32 e-12$ & $4.04 e-11$ \\
\hline & & $\left|x_{3}-\alpha\right|$ & $1.77 e-42$ & $1.67 e-42$ & $4.48 e-41$ & $2.90 e-39$ & $9.48 e-45$ & $4.52 e-41$ \\
\hline & & $\left|x_{4}-\alpha\right|$ & $1.01 e-166$ & $8.05 e-167$ & $1.84 e-160$ & $2.05 e-153$ & $4.77 e-176$ & $7.12 e-161$ \\
\hline & & $\left|x_{5}-\alpha\right|$ & $0 . e-299$ & $0 . e-299$ & $0 . e-299$ & $0 . e-299$ & $0 . e-299$ & $0 . e-299$ \\
\hline
\end{tabular}

${ }^{\dagger} 5.37 e-7=5.37 \times 10^{-7}$.

changes can occur as a result of rounding process in computing coefficients. Minimal round-off errors may improve the root finding of ill-conditioned problems. Certainly multi-precision arithmetic should be used in conjunction with optimized algorithms reducing round-off errors. Highorder methods with the asymptotic error constant of small magnitude are preferred for locating zeros with relatively good accuracy. Locating zeros for ill-conditioned problems is generally believed to be a difficult task.
It is also important to properly choose close initial values near the root for guaranteed convergence of the proposed method. Indeed, initial values are chaotic [23] to the convergence of the root $\alpha$. The following statement quoted from [24] is not too much to emphasize the importance of selected initial values: "A point that belongs to the non-convergent region for a particular value of the parameter can be in the convergent region for another parameter value, even though the former might have a higher order 
of convergence than the second. This then indicates that showing whether a method is better than the other should not be done through solving a function from a randomly chosen initial point and comparing the number of iterations needed to converge to a root."

Since our current analysis aims on the convergence of the proposed method, initial values [25-27] are selected in a small neighborhood of $\alpha$ for guaranteed convergence. Thus the chaotic behavior of $x_{0}$ on the convergence should be separately treated under the different subject in future analysis. On the one hand, future research may be more strengthened with the graphical analysis on the convergence including chaotic fractal basins of attractions. On the other hand, rational approximations [1] provide rich resources of future research on developing new high-order optimal methods for multiple zeros.

\section{Acknowledgments}

The first author (Y. I. Kim) was supported by the Research Fund of Dankook University in 2011; the corresponding author (Y. H. Geum) was also supported by the National Research Foundation of Korea funded by the Ministry of Education, Science, and Technology (Project No. 20110014638). In addition, the authors would like to give special thanks to anonymous referees for their valuable suggestions and comments on this paper.

\section{References}

[1] A. Iliev and N. Kyurkchiev, Nontrivial Methods in Numerical Analysis (Selected Topics in Numerical Analysis), Lambert Academic, Saarbrucken, Germany, 2010.

[2] J. F. Traub, Iterative Methods for the Solution of Equations, Prentice-Hall Series in Automatic Computation, Prentice-Hall, Englewood Cliffs, NJ, USA, 1964.

[3] $\mathrm{X}$. $\mathrm{Wu}$ and $\mathrm{D} . \mathrm{Fu}$, "New high-order convergence iteration methods without employing derivatives for solving nonlinear equations," Computers \& Mathematics with Applications, vol. 41, no. 3-4, pp. 489-495, 2001.

[4] B. I. Yun, "Iterative methods for solving nonlinear equations with finitely many roots in an interval," Journal of Computational and Applied Mathematics, vol. 236, no. 13, pp. 3308-3318, 2012.

[5] B. I. Yun, "A derivative free iterative method for finding multiple roots of nonlinear equations," Applied Mathematics Letters, vol. 22, no. 12, pp. 1859-1863, 2009.

[6] L. Atanassova, N. Kjurkchiev, and A. Andreev, "Two-sided multipointmethods of high order for solution of nonlinear equations, numerical methods and applications," in Proceedings of the International Conference on Numerical Methods and Applications, pp. 33-37, Sofia, Bulgaria, August 1989.

[7] C. Dong, "A family of multipoint iterative functions for finding multiple roots of equations," International Journal of Computer Mathematics, vol. 21, pp. 363-367, 1987.

[8] S. G. Li, L. Z. Cheng, and B. Neta, "Some fourth-order nonlinear solvers with closed formulae for multiple roots," Computers \& Mathematics with Applications, vol. 59, no. 1, pp. 126-135, 2010.

[9] X. Li, C. Mu, J. Ma, and L. Hou, "Fifth-order iterative method for finding multiple roots of nonlinear equations," Numerical Algorithms, vol. 57, no. 3, pp. 389-398, 2011.
[10] M. S. Petković, L. D. Petković, and J. Džunić, "Accelerating generators of iterative methods for finding multiple roots of nonlinear equations," Computers \& Mathematics with Applications, vol. 59, no. 8, pp. 2784-2793, 2010.

[11] J. R. Sharma and R. Sharma, "Modified Chebyshev-Halley type method and its variants for computing multiple roots," Numerical Algorithms, vol. 61, no. 4, pp. 567-578, 2012.

[12] X. Zhou, X. Chen, and Y. Song, "Constructing higher-order methods for obtaining the multiple roots of nonlinear equations," Journal of Computational and Applied Mathematics, vol. 235, no. 14, pp. 4199-4206, 2011.

[13] B. Neta and A. N. Johnson, "High-order nonlinear solver for multiple roots," Computers \& Mathematics with Applications, vol. 55, no. 9, pp. 2012-2017, 2008.

[14] B. Neta, "Extension of Murakami's high-order non-linear solver to multiple roots," International Journal of Computer Mathematics, vol. 87, no. 5, pp. 1023-1031, 2010.

[15] T. Murakami, "Some fifth order multipoint iterative formulae for solving equations," Journal of Information Processing, vol. 1, pp. 138-139, 1978.

[16] L. Shengguo, L. Xiangke, and C. Lizhi, "A new fourth-order iterative method for finding multiple roots of nonlinear equations," Applied Mathematics and Computation, vol. 215, no. 3, pp. 12881292, 2009.

[17] P. Jarratt, "Some efficient fourth order multipoint methods for solving equations," BIT Numerical Mathematics, vol. 9, pp. 119124, 1969.

[18] J. R. Sharma and R. Sharma, "Modified Jarratt method for computing multiple roots," Applied Mathematics and Computation, vol. 217, no. 2, pp. 878-881, 2010.

[19] B. Neta, Numerical Methods for the Solution of Equations, NetA-Sof, Calif, USA, 1983.

[20] R. Sharma, "Some more inequalities for arithmetic mean, harmonic mean and variance," Journal of Mathematical Inequalities, vol. 2, no. 1, pp. 109-114, 2008.

[21] H. T. Kung and J. F. Traub, "Optimal order of one-point and multipoint iteration," Journal of the Association for Computing Machinery, vol. 21, pp. 643-651, 1974.

[22] S. Wolfram, The Mathematica Book, Wolfram Media, Champaign, Ill, USA, 4th edition, 1999.

[23] D. Gulick, Encounters with Chaos, Prentice-Hall, New York, NY, USA, 1992.

[24] H. Susanto and N. Karjanto, "Newton's method's basins of attraction revisited," Applied Mathematics and Computation, vol. 215, no. 3, pp. 1084-1090, 2009.

[25] F. Soleymani and S. Shateyi, "Two optimal eighth-order derivative-free classes of iterative methods," Abstract and Applied Analysis, vol. 2012, Article ID 318165, 14 pages, 2012.

[26] F. Soleymani, S. Shateyi, and H. Salmani, "Computing simple roots by an optimal sixteenth-order class," Journal of Applied Mathematics, vol. 2012, Article ID 958020, 13 pages, 2012.

[27] F. Soleymani, D. K. R. Babajee, S. Shateyi, and S. S. Motsa, "Construction of optimal derivative-free techniques without memory," Journal of Applied Mathematics, vol. 2012, Article ID 497023, 24 pages, 2012. 


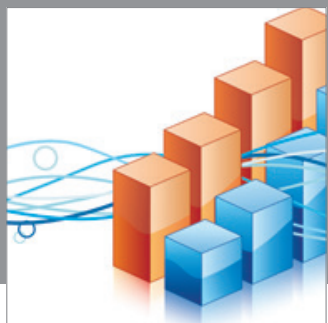

Advances in

Operations Research

mansans

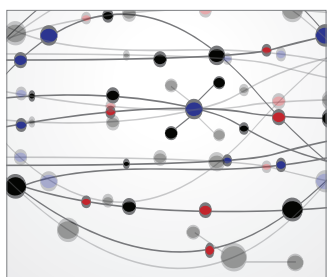

The Scientific World Journal
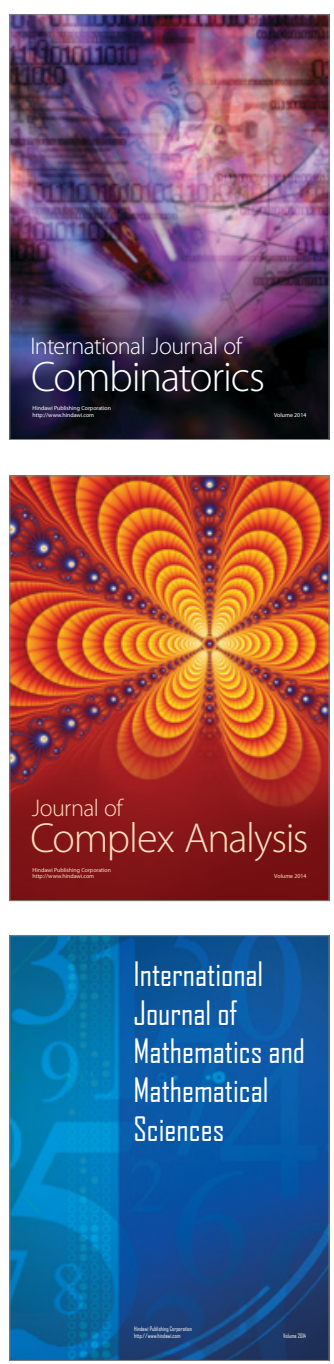
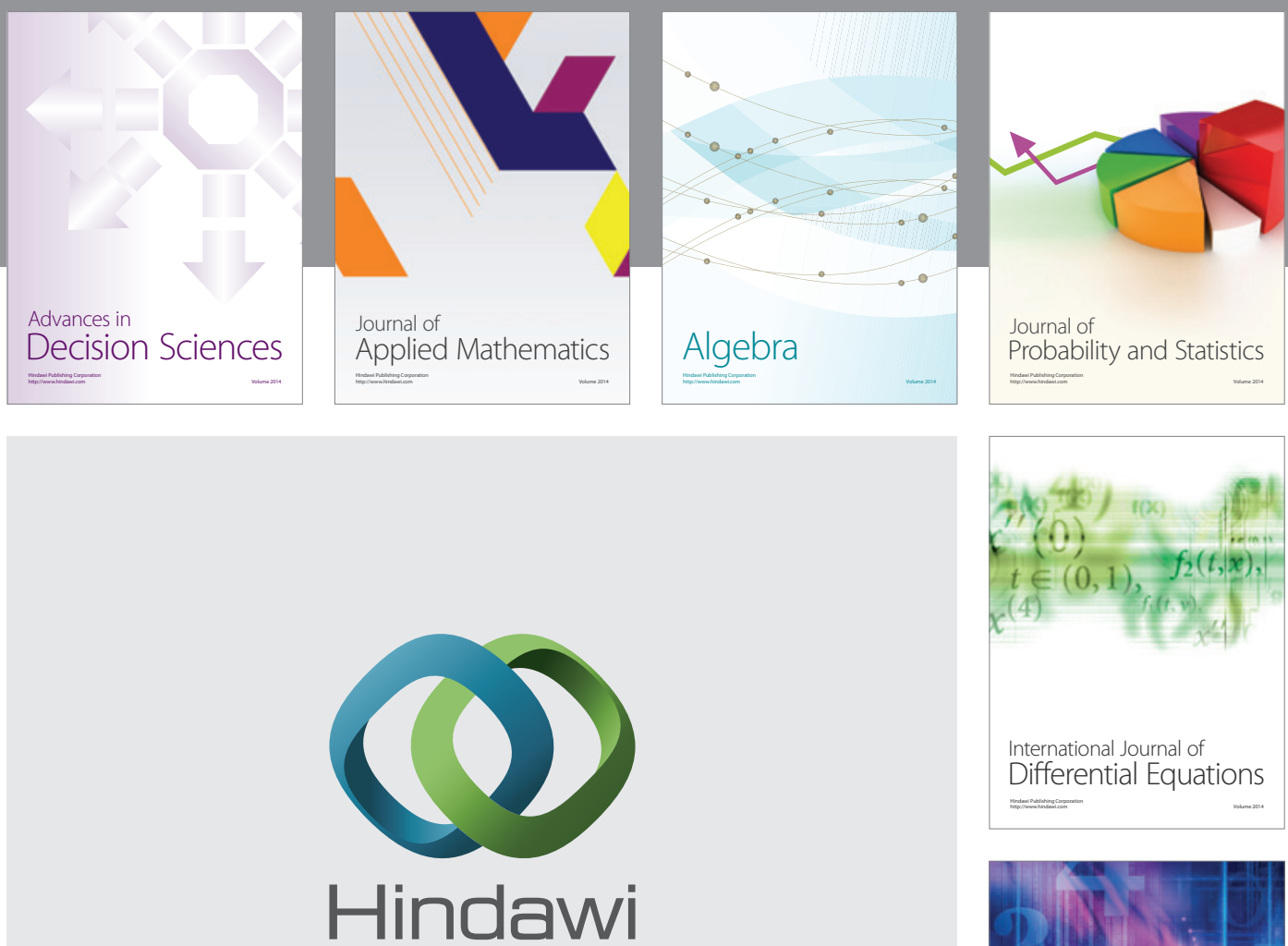

Submit your manuscripts at http://www.hindawi.com
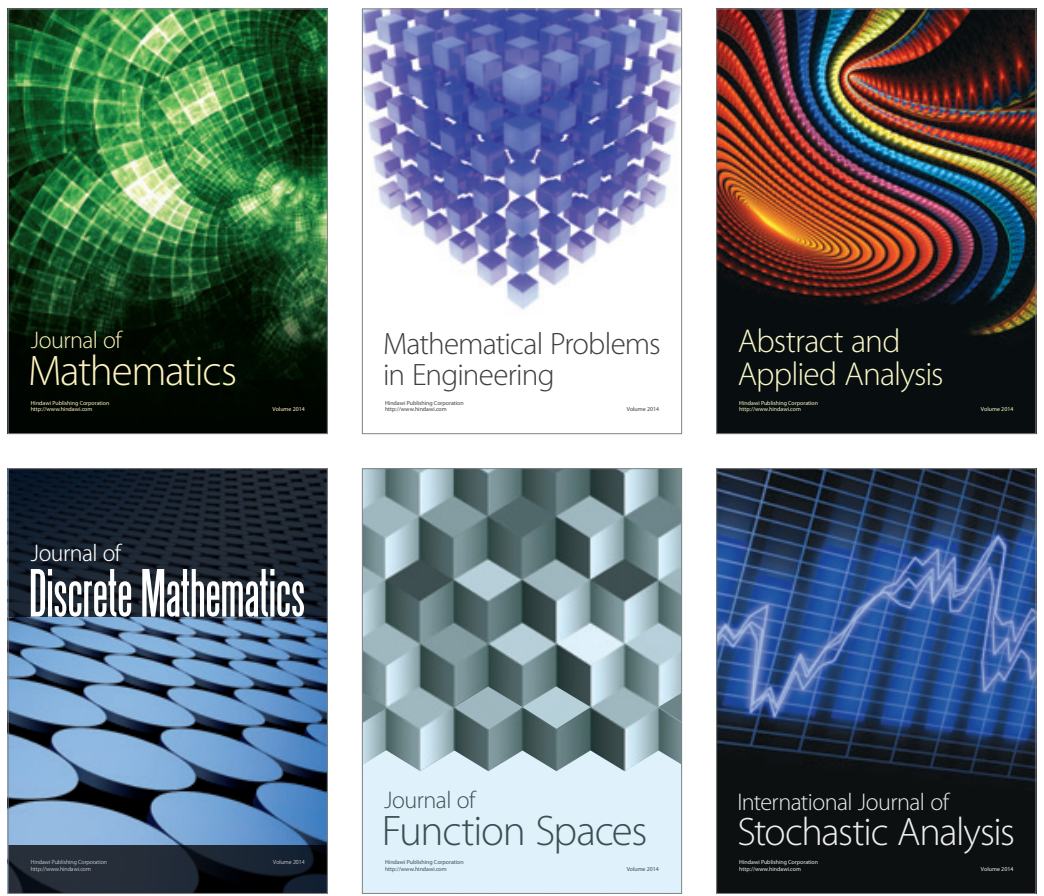

Journal of

Function Spaces

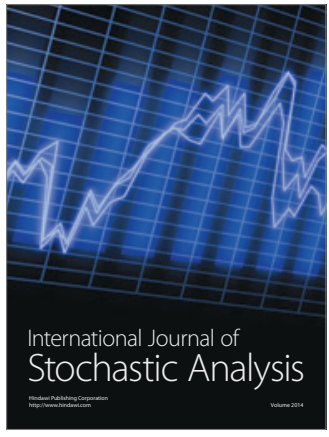

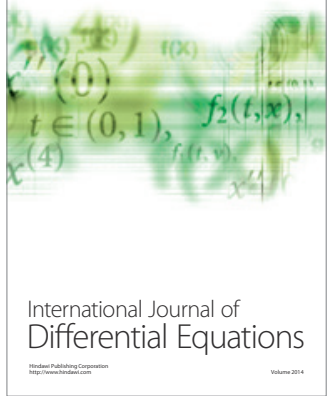
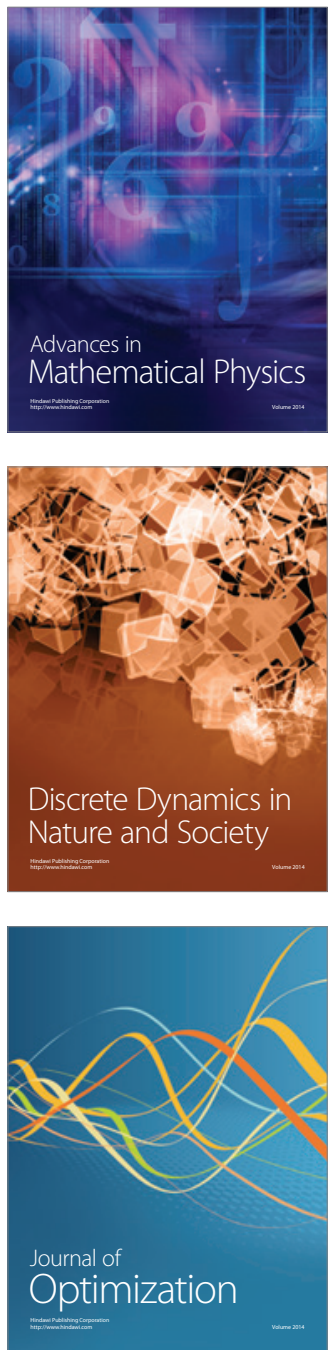